\title{
Towards an accurate determination of the critical exponents with the Renormalization Group flow equations
}

\author{
A. Bonanno \\ Osservatorio Astrofisico \\ Via S.Sofia 78, I-95138, Catania \\ INFN, Sezione di Catania \\ Corso Italia 57, I-95129, Catania, Italy \\ and \\ D. Zappalà \\ INFN, Sezione di Catania \\ Dipartimento di Fisica, Università di Catania \\ Corso Italia 57, I-95129, Catania, Italy
}

\begin{abstract}
The determination of the critical exponents by means of the Exact Renormalizion Group approach is still a topic of debate. The general flow equation is by construction scheme independent, but the use of the truncated derivative expansion generates a model dependence in the determination of the universal quantities. We derive new nonperturbative flow equations for the one-component, $Z_{2}$ symmetric scalar field to the next-to-leading order of the derivative expansion by means of a class of proper time regulators. The critical exponents $\eta, \nu$ and $\omega$ for the Wilson-Fisher fixed point are computed by numerical integration of the flow equations, without resorting to polynomial truncations. We show that by reducing the width of the cut-off employed, the critical exponents become rapidly insensitive to the cut-off width and their values are in good agreement with the results of entirely different approaches.
\end{abstract}

Pacs 11.10.Hi , 11.10.Kk 
Universality is the property which guarantees that, at a critical point, the divergences of the correlation functions of an entire class of physical systems have the same power law behavior with the same exponents (critical exponents), regardless of their specific features and especially of their microscopic (ultraviolet $(\mathrm{UV})$ ) details. This notion is very general and it often appears in the study of critical phenomena and in quantum field theory. The prediction of those exponents has been recognized to be one of the most important achievements of the renormalization group approach pioneered by Wilson and others [1, 2]. A standard approach to this problem is the $\epsilon$-expansion [2] method, whose main feature is an expansion in $\epsilon=4-D$ (where $D$ indicates the number of dimensions) of the renormalized free energy. Unfortunately this method is not entirely satisfactory, since actual values for three-dimensional systems are obtained only after setting $\epsilon=1$ at the end of the calculation. On the other hand, ordinary perturbation theory in such nonperturbative framework requires quite involved loop calculations [3]. Moreover since both $\epsilon$-expansion and perturbative expansion at fixed dimension yield divergent series, one has to rely on the Borel summability of these series in order to get meaningful results.

In recent years the Exact Renormalization Group (ERG) approach has proven to be very useful in many situations where the usual perturbative methods are not totally satisfactory [4, 5]. The main step in this approach is to solve the flow equation which is a complicate non-linear functional equation. For this reason one must then resort to sofisticate approximation methods in order to extract physical information. The derivative expansion allows a systematic expansion of the renormalized action in terms of local field operators. It has the advantage of recasting the functional flow equation into a set of coupled partial non-linear differential equations for the local potential $U_{k}(\phi)$ and for the wavefunction renormalization function $Z_{k}(\phi)$ in the next-to-leading order approximation which improves the so called Local Potential Approximation (LPA) where only the equation for $U_{k}(\phi)$ is considered and $Z_{k}(\phi)=1$ is kept fixed. This topic has been the subject of an intense investigation and the reader is referred to the excellent review in [6] for a recent survey of results for the scalar theory. Although, as far as the calculation of universal quantities is concerned, the exact evolution equation is by construction scheme independent, this no longer holds in specific truncations of the full evolution equation. In fact different realizations of the smooth cut-off ERG equations determine critical exponents which are different if, for instance, an exponential cut-off [7] or a power law cut-off is used [8, 9].

Recently, the ERG scheme dependence has been the subject of many papers. In [10] the scheme dependence has been analyzed in order to optimize the convergence of approximate solutions of the ERG equations. Other authors have considered the effects of the scheme dependence in the convergence of polynomial 
truncations of the flow equation in the LPA [11, 12]. Actually it would be desirable to have an approximation framework which allows to control the scheme dependence to each order in the derivative expansion, without resorting to further polynomial truncations and which, at the same time, could provide an estimate of the uncertainty on the determination of the universal quantities.

The first determination of the critical exponents $\nu, \omega$ from a numerical resolution of the flow equation has been performed in [13] by using the LPA of the Wegner-Houghton (WH) equation for the wilsonian action, 14, which is a sharp cut-off version of the ERG equation. However the WH equation, truncated to the next-to-leading order in the derivative expansion although predicts the correct two loop value of the field anomalous dimension $\eta$ in $D=4$ [15], fails to give a reasonable value of $\eta$ at the Wilson-Fisher fixed point in $D=3$ [16].

To overcome the difficulties with the sharp cut-off formulation, several authors [17, 18, 19 have proposed a smooth cut-off realization of the ERG equations by means of the Schwinger proper time regulator. This approach has been used in many contexts like Yang-Mills theories [20 and Chiral Symmetry Breaking [12, 21]. In [12 the effect of this regulator on specific polynomial truncations of the LPA has been discussed. In [22 the same cut-off function is used to include the effect of a field independent wavefunction renormalization function $Z_{k}$ in the $O(N)$ model. However the inclusion of the field dependence in $Z_{k}$ turns out to be essential for a consistent determination of the scaling properties of the system. A discussion on the determination of the wavefunction renormalization function within this framework has been presented even in [19], but so far the full flow equation for $Z_{k}(\phi)$ within this approach has not been derived.

The aim of this paper is to derive the full coupled flow equations for $Z_{k}(\phi)$ and $U_{k}(\phi)$ by means of the general class of proper time regulators introduced in [17, 18, 19] and to numerically determine the anomalous dimension $\eta$ and the critical exponents $\nu$ and $\omega$, by solving these equations around the Wilson-Fisher fixed point in $D=3$. We also show that, when diminishing the size of the cut-off width, the critical exponents become stable, converging to fixed values.

Let us now review the basic assumptions in the derivation of our evolution equation. The one-loop contribution to the effective action is given by the trace of $\log \left(\delta^{2} S /(\delta \phi \delta \phi)\right)$, where $S$ is the classical action. By introducing the Schwinger proper time representation for the logarithm with the proper time variable $s$, we can express the one-loop contribution as

$$
S_{k}^{1-l o o p}=-\frac{1}{2} \operatorname{Tr} \int_{0}^{\infty} \frac{d s}{s} f_{k} \exp \left(-s \frac{\delta^{2} S}{\delta \phi \delta \phi}\right)
$$

where we have introduced the heat-kernel smooth regulator $f_{k}$. The usual one- 
loop effective action is recovered by setting $f_{k}=1$ and properly regulating the UV divergences in Eq. (11).

In the spirit of the derivation of the $\mathrm{WH}$ equation, the flow of the wilsonian action is obtained by taking $f_{k}$ as a sharp momentum cut-off, i.e. a Heaviside function which is equal to one for momenta greater than $k$ and vanishes for momenta smaller than $k$, and then by performing the integration of the modes of the action through infinitesimal steps $\delta k$ in the momentum space. Therefore the action $S_{k-\delta k}$ comes from the integration of the modes between $k-\delta k$ and $k$ in the action $S_{k}$ defined at the scale $k$. A smooth cut-off on the momenta in this framework is then obtained by choosing a non-singular $f_{k}$ instead of the Heaviside function and the differential flow equation reads

$$
k \frac{\partial S_{k}}{\partial k}=-\frac{1}{2} \operatorname{Tr} \int_{0}^{\infty} \frac{d s}{s} k \frac{\partial f_{k}}{\partial k} \exp \left(-s \frac{\delta^{2} S_{k}}{\delta \phi \delta \phi}\right)
$$

It must be remarked that the WH equation contains terms that are neglected in Eq. (2) but, as discussed in [23], these terms do not contribute to the next-toleading truncation in the derivative expansion.

About $f_{k}$ one can in general require the following properties (see [18, 19]): 1) $f_{k}$ must depend on $k$ through the dimensionless quantity $s k^{2}$. We shall consider, as in 22 the variable $s Z_{k} k^{2}$, where $Z_{k}$ is the wave function renormalization function, in order to reproduce the usual form of the propagator in the following equations. 2) $f_{k=0}=1$ so that $S_{k=0}$ must be identified with the full effective action. This implies that in the limit $k \rightarrow 0$ the infrared cut-off is removed. 3) $f_{k}\left(s Z_{k} k^{2}\right)$ must tend to zero sufficiently rapidly for large $s Z_{k} k^{2}$ to suppress the small momentum modes. In particular we choose, for any integer $m \geq 1$

$$
f_{k}^{(m)}\left(s Z_{k} k^{2}\right)=e^{-s Z_{k} k^{2}} \sum_{i=0}^{m} \frac{\left(s Z_{k} k^{2}\right)^{i}}{i !}
$$

We note that, as it stands, Eq. (2) for some values of $m$ and of the dimension $D$ is not UV convergent, but in $D=3$ and for $m \geq 1$, which is the case we are interested in, no UV divergence appear in the differential flow equation.

An analysis of the polynomial truncations of the LPA to Eq. (2) for $m=1,2,3$ has been performed in [12], where it is shown that all the parameters extracted from the flow equations, regarded as functions of the polynomial truncation, converge more rapidly when $m$ grows. This is a first indication that the RG equations provide more stable results for increasing values of $m$.

As anticipated above, we shall focus here on the first order truncation in the derivative expansion of the action $S_{k}$ which corresponds to the following 
parametrization

$$
S_{k}=\int d^{D} x\left(\frac{Z_{k}(\phi)}{2} \partial_{\mu} \phi \partial^{\mu} \phi+V_{k}(\phi)\right)
$$

and therefore Eq. (2) is reduced to two coupled partial differential equations for $V_{k}(\phi)$ and $Z_{k}(\phi)$. No further polynomial expansion or truncation in powers of the field $\phi$ is made on $V_{k}(\phi)$ and $Z_{k}(\phi)$.

In order to project the flow equation on the local potential term and on the kinetic term we first set (see also [24, 23, 15]) $\phi=\phi_{0}+\varphi(x)$ where $\phi_{0}$ is a constant field configuration and $\varphi(x)$ is a small fluctuation field. Next, we expand $V_{k}(\phi)$ and $Z_{k}(\phi)$ in both sides of Eq. (2) and retain only terms at most quadratic in $\partial \varphi$. Then we define $\delta^{2} S_{k} /(\delta \phi \delta \phi) \equiv A=A_{0}+\delta A$ where $A_{0}$ is only a function of the constant field $\phi_{0}$ and $\delta A$ contains all the linear and quadratic contributions in $\partial \varphi$. Thus the trace becomes

$$
\begin{aligned}
& -\frac{1}{2} \operatorname{Tr} \int_{0}^{\infty} \frac{d s}{s} k \frac{\partial f_{k}}{\partial k} e^{-s A}= \\
& \int d^{D} x \int_{0}^{\infty} d s Z_{k} k^{2} \frac{\left(s Z_{k} k^{2}\right)^{m}}{m !} e^{-s Z_{k} k^{2}}\left\langle x\left|e^{-s\left(A_{0}+\delta A\right)}\right| x\right\rangle= \\
& \int d^{D} x \int_{0}^{\infty} d s Z_{k} k^{2} \frac{\left(s Z_{k} k^{2}\right)^{m}}{m !} e^{-s Z_{k} k^{2}}\langle x| e^{-s A_{0}}(1-s \delta A \\
& \left.\left.+\frac{s^{2}}{2 !}\left\{\left[\delta A, A_{0}\right]+\delta A^{2}\right]\right\}+\ldots\right)|x\rangle
\end{aligned}
$$

where in order to disentangle the trace in Eq. (5) we used the Baker-CampbellHausdorff formula, and the dots stand for the higher order terms in the $s$ expansion of the exponential. Actually, in order to collect all the contributions to the wavefunction renormalization function we had to retain terms up to the $O\left(s^{4}\right)$ order in this expansion. The evaluation of the trace, as explained in [24, 23, 15], is then performed by inserting the identity $\int d p^{D}|p\rangle\langle p|$ in the trace in Eq. (5) and by making use of the commutation relation $\left[p_{\mu}, f(x)\right]=i \partial_{\mu} f(x)$. Finally, by collecting on both sides of Eq. (5) the coordinate independent terms and the coefficients of the kinetic term $\partial \varphi \partial \varphi$, we get respectively the flow equation for $V_{k}$ and for $Z_{k}$. Remarkably, all the integrals in the proper time $s$ can be performed analytically and therefore we end up not with integrodifferential flow equations, but simply with differential equations.

It is convenient to express the flow equations in terms of new dimensionless quantities $t, \Phi, V(t, \Phi), Z(t, \Phi)$, defined by the following relations: $t=$ $\ln (\Lambda / k), \quad \phi=k^{(1+\eta) / 2} \sqrt{\alpha} \Phi, \quad V_{k}(k, \phi)=\alpha k^{3} V(t, \Phi)$ and $Z_{k}(k, \phi)=k^{-\eta} Z(t, \Phi)$ where $\eta$ is the anomalous dimension of $\phi, \Lambda$ is a UV reference momentum scale, and the constant $\alpha$ is defined in terms of the Gamma functions: $\alpha=(\Gamma(m-$ 
$1 / 2)) /(8 \pi \sqrt{\pi} m !))$. The flow equations then read (the / indicates derivative w.r.t. the field $\Phi)$

$$
\begin{gathered}
\frac{\partial V}{\partial t}=3 V-\frac{1+\eta}{2} \Phi V^{\prime}-\left(\frac{Z}{Z+V^{\prime \prime}}\right)^{m-1 / 2} \\
\frac{\partial Z}{\partial t}=-\eta Z-\frac{1+\eta}{2} \Phi Z^{\prime}+\left(\frac{Z}{Z+V^{\prime \prime}}\right)^{m-1 / 2}\left(\frac{(m-1 / 2)}{Z+V^{\prime \prime}}\left(Z^{\prime \prime}-\frac{49\left(Z^{\prime}\right)^{2}}{24 Z}\right)-\right. \\
\left.\frac{7\left(m^{2}-1 / 4\right)}{6\left(Z+V^{\prime \prime}\right)^{2}} Z^{\prime} V^{\prime \prime \prime}+\frac{\left(m^{2}-1 / 4\right)(m+3 / 2)}{6\left(Z+V^{\prime \prime}\right)^{3}} Z\left(V^{\prime \prime \prime}\right)^{2}\right)
\end{gathered}
$$

In order to determine the critical exponents we need to find the fixed points of Eqs. (6,7) and then to study the linearized version of the equations around the fixed point solutions. The latter are the $t$-independent solutions of Eqs. (6, (7). They are indicated as $V^{*}(\Phi)$ and $Z^{*}(\Phi)$. Once $V^{*}(\Phi)$ and $Z^{*}(\Phi)$ are determined, one can linearize Eqs. (6, (7), by introducing small $t$-dependent perturbations around $V^{*}(\Phi)$ and $Z^{*}(\Phi)$ :

$$
\begin{aligned}
& V(t, \Phi)=V^{*}(\Phi)+\delta V(t, \Phi)=V^{*}(\Phi)+e^{\lambda t} v(\Phi) \\
& Z(t, \Phi)=Z^{*}(\Phi)+\delta Z(t, \Phi)=Z^{*}(\Phi)+e^{\lambda t} z(\Phi)
\end{aligned}
$$

and by retaining in Eqs. (6,7) only linear terms in $v(\Phi), z(\Phi)$.

It is worthwhile to notice that Eqs. (6,7) are invariant under the transformations $\Phi \rightarrow \epsilon \Phi, Z \rightarrow \epsilon^{-2} Z$. This is the consequence of a reparametrization invariance of Eq. (2) where $\phi$ and $Z_{k}$ transform as $\Phi$ and $Z$ respectively and the proper time is redefined as $s \rightarrow \epsilon^{2} s$, and all the other quantities are not modified.

Before going on we consider in more detail the behavior of the regulator $f_{k}$ when $m$ grows. As a simple check one can go back to Eq. (5) and neglect the $Z_{k}$ effects. In this case $\delta A=0$ and no expansion of the exponential is needed and only the term $\exp \left(-s A_{0}\right)$ is left. By integrating the variable $s$ we get

$$
\begin{aligned}
-\frac{1}{2} \operatorname{Tr} \int_{0}^{\infty} \frac{d s}{s} k \frac{\partial f_{k}}{\partial k} \quad e^{-s A_{0}} & =\int d^{3} p \int_{0}^{\infty} d s k^{2} \frac{\left(s k^{2}\right)^{m}}{m !} e^{-s\left(k^{2}+A_{0}\right)}= \\
& 4 \pi \int_{0}^{\infty} d p p^{2}\left(\frac{k^{2}}{k^{2}+p^{2}+V_{k}^{\prime \prime}}\right)^{m+1}
\end{aligned}
$$

Clearly the $p$ integration leads back to Eq. (6) with $Z=1$, but from the integrand in the r.h.s. of Eq. (9) it is possible to learn which momenta $p$ are relevant in the integration. In Fig. 1 the rescaled integrand $F(p)=m p^{2}\left(k^{2} /\left(k^{2}+\right.\right.$ 
$\left.\left.p^{2}+V_{k}{ }^{\prime \prime}\right)\right)^{m+1}$ for some values of $m$ and for fixed $k$ is plotted. The maximum of $F(p)$ is at $\bar{p}^{2}=\left(k^{2}+V_{k}{ }^{\prime \prime}\right) / m$ and $F(\bar{p})=\left(k^{2}+V_{k}{ }^{\prime \prime}\right)^{-m}\left(m k^{2} /(m+1)\right)^{m+1}$. So, when $m$ grows, the cut-off $f_{k}$ has the effect of selecting smaller and smaller momentum shells, centered at $\bar{p}$ where the integrand behaves as a power of the propagator. Then, the large $m$ limit is a kind of sharp cut-off limit for a modified propagator, which however cannot be directly related to the sharp cut-off $\mathrm{WH}$ equation. Indeed the logarithmic behavior of the latter is clearly different from the one of Eq. (6) where the term $\left(Z /\left(Z+V^{\prime \prime}\right)\right)^{m-1 / 2}$ appears instead. At the same time no straightforward limit $m \rightarrow \infty$ can be taken in Eqs. (6, (7) in order to determine the analytic asymptotic behavior of the critical exponents. This can be seen in the LPA from Eq. (河) with $Z=1$. In this case, when $m \rightarrow \infty$, the sign of $V^{\prime \prime}$ determines whether the last term in (6) diverges or vanishes (or it is one for $V^{\prime \prime}=0$ ). Therefore no uniform convergence of the solution is found in the limit $m \rightarrow \infty$. Unfortunately, a deeper understanding of the relation between this heat-kernel regulator and the other formulations of the ERG equations is still missing and we have to limit ourselves to compare the numerical results of the different methods.

We are interested in determining the anomalous dimension $\eta$ at the only nongaussian fixed point solution, the Wilson-Fisher fixed point, and the exponents $\nu$ and $\omega$ which are related to the eigenvalues $\lambda$ of the linearized equations, being defined as the inverse of the only positive eigenvalue and as the opposite of the less negative eigenvalue respectively.

The first step concerns the determination of non-trivial solutions $V^{*}(\Phi)$ and $Z^{*}(\Phi)$ and we closely follow the procedure outlined in [9] (see also [16]). We use the shooting method in order to numerically solve the fixed point equations taking, as boundary conditions, the asymptotic expression of the potential and wave function renormalization function at large $\Phi$ together with the symmetry property of these functions at the origin: $Z^{* \prime}(0)=V^{* \prime}(0)=0$. The normalization at the origin $Z^{*}(0)$ must be fixed to some definite value and, as a test of the reparametrization invariance, we checked that the numerical results found for the critical exponents do not depend on the particular value of this normalization. The procedure to determine the asymptotic expressions from the equations is explained in detail in [9] and therefore we do not repeat it here. In practice, as it happens for the sharp cut-off equations, [16], we find that the numerical resolution of the problem is simpler if the equation for the potential is replaced by the corresponding equation for its field derivative $V^{* \prime}(\Phi)$. For each value of $m$ we find only one non-trivial fixed point as it was expected and the values of the anomalous dimension for each $m$ are displayed in Table 1.

Having determined the fixed point, we again use the shooting procedure to 


\begin{tabular}{|c|c||c|c|}
\hline$m$ & $\eta$ & $m$ & $\eta$ \\
\hline 1 & 0.0653 & 11 & 0.0365 \\
2 & 0.0507 & 12 & 0.0362 \\
3 & 0.0452 & 13 & 0.0360 \\
4 & 0.0423 & 14 & 0.0358 \\
5 & 0.0405 & 15 & 0.0356 \\
6 & 0.0393 & 16 & 0.0354 \\
7 & 0.0385 & 17 & 0.0353 \\
8 & 0.0378 & 20 & 0.0350 \\
9 & 0.0373 & 30 & 0.0343 \\
10 & 0.0369 & 40 & 0.0340 \\
\hline
\end{tabular}

Table 1: The anomalous dimension $\eta$ at the Wilson-Fisher fixed point determined for various values of $m$.

evaluate $\nu$ and $\omega$ from the linearized equations as explained in 9]. The results are reported in Table 2 as the $O\left(\partial^{2}\right)$ approximation estimates. In order to test the improvement due to the inclusion of the wave function renormalization function, we also show in Table 2 the values of $\nu, \omega$ obtained within the LPA $\left(O\left(\partial^{0}\right)\right)$, i.e. from the linearized potential equation with $\eta=0$ and $Z=1$ fixed.

We note that the numerical procedure employed to solve the equations becomes more problematical and converges less rapidly for larger values of $m$. Moreover, since the fixed point equations are more stable than the linearized ones we can push $m$ up to $m=40$ in the former case but only up to $m=9$ in the latter case.

\begin{tabular}{|c||c|c||c|c||}
\hline \multicolumn{1}{|c||}{$m$} & \multicolumn{2}{c||}{$\nu$} & \multicolumn{2}{c||}{$\omega$} \\
\hline & $O\left(\partial^{0}\right)$ & $O\left(\partial^{2}\right)$ & $O\left(\partial^{0}\right)$ & $O\left(\partial^{2}\right)$ \\
\hline 1 & 0.6604 & 0.6348 & 0.629 & 0.847 \\
2 & 0.6439 & 0.6331 & 0.674 & 0.762 \\
3 & 0.6381 & 0.6311 & 0.698 & 0.738 \\
4 & 0.6351 & 0.6299 & 0.711 & 0.727 \\
5 & 0.6333 & 0.6290 & 0.720 & 0.721 \\
6 & 0.6322 & 0.6286 & 0.727 & 0.717 \\
7 & 0.6313 & 0.6279 & 0.731 & 0.713 \\
8 & 0.6306 & 0.6277 & 0.735 & 0.711 \\
9 & 0.6301 & 0.6276 & 0.738 & 0.710 \\
\hline
\end{tabular}

Table 2: Values of the exponents $\nu, \omega$ for various $m$. The $O\left(\partial^{0}\right)$ (LPA) values are obtained from the potential equation only with $Z=1, \eta=0$ fixed. 
The first thing that should be noted is that the change of $\eta, \nu, \omega$ diminishes when $m$ grows and the value of these parameters becomes almost constant : $\nu$ and $\omega\left(O\left(\partial^{2}\right)\right)$ already at $m=9$ are almost stable up to the third significant digit and, according to the trend shown up to $m=40$, a rapid convergence of $\eta$ is observed. The dependence on the parameter $m$ is practically vanishing. Since the analytical approach to the $m \rightarrow \infty$ limit of Eqs. (6, (7) is troublesome, we have to rely on the numerical results shown in Tables 1,2 in order to understand the behavior of $\eta, \nu, \omega$ for large values of $m$. As an additional check we have considered a fit to the numbers in Table 1 with various analytical trial forms of the function $\eta(m)$, containing a few free parameters. In these checks the $\chi$-square analysis clearly indicates a finite asymptotic value of $\eta(m \rightarrow \infty)$ around $\eta=0.0329$.

As far as the derivative expansion improvement is concerned, $\nu$ and $\omega$ behave differently in the two approximations here considered: the former is always decreasing with $m$; the latter has a different trend in the two approximations and the LPA gives a better estimate than the $O\left(\partial^{2}\right)$ approximation. However it must be remarked that both $\nu, \omega$ become more stable with $m$ when both equations for $v$ and $z$ are considered, rather than in the LPA. It is conceivable to expect that, when going to higher orders in the derivative expansion, the critical exponents converge to a value that sits between the $O\left(\partial^{0}\right)$ and the $O\left(\partial^{2}\right)$ estimates.

In Table 3 we report some estimates of the critical exponents obtained by various formulations of the ERG equations and by completely different methods (see captions for references). Table 3 collects only few relevant results and it is by far an incomplete list. Much more exhaustive lists can be found in [3, 5, 6, 33]. A comparison with the results in Tables 1 and 2 indicates that the particular cut-off here employed provides a little improvement in the agreement with the non-ERG approaches, on the anomalous dimension determination. Concerning $\nu$ and $\omega$ our determinations are within the average, but slightly smaller (although it should be noticed the small experimental value of $\nu$ ).

Therefore, the convergence of the numerical results in Tables 1 and 2 to such good values in the limit of large $m$, provides an indication that the heat-kernel cut-off, in that limit, becomes a sensible regulator of both ultraviolet and infrared modes. Moreover it is particularly effective in reducing the weight of all the irrelevant operators neglected in our derivative expansion truncation, which, on the other hand, as it is argued in [1], become important for the same truncation of the WH equation.

In conclusion, we have presented a particular version of the RG equations for a single field scalar theory with a heat-kernel cut-off, introduced in the Schwinger proper time formalism. This cut-off is parametrized by an index that regulates the size of the momentum shell integrated in the blocking procedure. We con- 


\begin{tabular}{|c|c|c|c|}
\hline & $\eta$ & $\nu$ & $\omega$ \\
\hline (a) & 0.0467 & 0.6307 & \\
(b) & 0.0539 & 0.6181 & 0.897 \\
(c) & $0.040(7)$ & $0.626(9)$ & $0.85(7)$ \\
(d) & 0.042 & 0.622 & 0.754 \\
\hline \hline (e) & $0.0335(25)$ & $0.6304(13)$ & $0.799(11)$ \\
(f) & $0.0360(50)$ & $0.6290(25)$ & $0.814(18)$ \\
(g) & & $0.6300(15)$ & $0.825(50)$ \\
(h) & & $0.6310(5)$ & \\
(i) & $0.026(3)$ & $0.624(2)$ & $0.82(3)$ \\
(l) & $0.0374(14)$ & $0.6294(9)$ & $0.87(10)$ \\
\hline \hline (p) & & $0.625(6)$ & $0.80(5)$ \\
\hline
\end{tabular}

Table 3: Values of the exponents $\eta, \nu, \omega$, calculated with various methods.

(a) $R G$ effective average action approach with exponential smooth cut-off, to $O\left(\partial^{2}\right)$ order [5].

(b) ERGE with powerlike smooth cut-off to $O\left(\partial^{2}\right)$ order [9, 25].

(c) ERGE expansion into a truncated set of ordinary differential equation [20], quoted from [6].

(d) Polchinski version of the ERGE [2X].

(e) Seven-loop perturbation series in $D=3$ [3].

(f) Five-loop $\epsilon$-expansion [3].

(g) high temperature series [28], quoted from [3].

(h) high temperature series [29], quoted from [3].

(i) Monte Carlo simulation [30].

(l) Monte Carlo simulation [31, quoted from [3].

(p) experimental data from the liquid-vapour transition. quoted from 39.

sidered a derivative expansion of the flow equation truncated to the $O\left(\partial^{2}\right)$ order, obtaining a set of two coupled equations which are reparametrization invariant. The corresponding determination of $\eta, \nu, \omega$ at the Wilson-Fisher fixed point in three dimensions is substantially encouraging due to the good agreement with the other estimates of these parameters.

We would like to acknowledge Martin Reuter for very enlightening discussions and encouragements. 


\section{References}

[1] L.P. Kadanoff, Physica 2, (1966), 263. K.G. Wilson, Phys. Rev. B4, (1971), 3174 and 3184.

[2] K.G. Wilson and M.E. Fisher, Phys. Rev. Lett. 28, (1972), 240; K.G. Wilson and J. Kogut, Phys. Rep. 12, (1974), 75.

[3] R. Guida and J. Zinn-Justin, J. Phys. A 31, (1998), 8103.

[4] J. Berges, Lectures at the 11th Summer School and Symposium on Nuclear Physics (NuSS 98): Effective Theories of Matter (1), Seoul, Korea, 23-27 Jun 1998, hep-ph/9902419.

[5] J. Berges, N. Tetradis and C. Wetterich, Nonperturbative renormalization group flow in quantum field theory and statistical physics, Preprint: MITCTP-2980, HD-THEP-00-26, May 2000 and hep-ph/0005122. Submitted to Phys.Rep.

[6] C. Bagnuls and C. Bervillier, Exact renormalization group equations. An introductory review, Preprint: SACLAY-SPHT-T00-008, SACLAY-SPHTS00-009, Feb 2000 and hep-th/0002034. Submitted to Phys.Rep.

[7] C. Wetterich, Nucl. Phys. B352, (1991), 529; Z. Phys. C57, (1993), 451; C60 (1993) 461; Phys Lett. B301, (1993), 90.

[8] T. Morris, Int. J. Mod. Phys. A 9 (1994), 2411.

[9] T. Morris, Phys. Lett. B329, (1994), 241.

[10] D.F. Litim, Phys.Lett. B486, (2000) 92.

[11] S.-B. Liao, J. Polonyi and M. Strickland, Nucl. Phys. B567, (2000), 493.

[12] G. Papp, B.J. Schaefer, H.J. Pirner and J. Wambach, Phys. Rev. D61 (2000) 096002.

[13] A. Hasenfratz and P. Hasenfratz, Nucl. Phys. B270, (1986) 687.

[14] F. J. Wegner and A. Houghton, Phys. Rev A8, (1972) 401.

[15] A. Bonanno and D. Zappalà, Phys. Rev. D57, (1998), 7383.

[16] A. Bonanno, V. Branchina, H. Mohrbach and D. Zappalà, Phys. Rev. D60, (1999), 065009. 
[17] M. Oleszczuk, Z. Phys. C64, (1994), 533.

[18] R. Floreanini and R. Percacci, Phys. Lett. B356, (1995), 205.

[19] S.-B. Liao, Phys. Rev. D53, (1996), 2020.

[20] S.-B. Liao, Phys. Rev. D56, (1997), 5008.

[21] B.J. Schaefer and H.J. Pirner, Nucl. Phys. A627, (1997), 481; Nucl. Phys. A660 (1999) 439; J. Meyer, G. Papp, H.J. Pirner and T. Kunihiro, Phys. Rev. C61, (2000), 035202.

[22] O. Bohr, B.J. Schaefer and J. Wambach, Renormalization group flow equations and the phase transition in $O(N)$ models, Preprint July 2000. e-Print Archive: hep-ph/0007098.

[23] S.-B. Liao and J. Polonyi, Phys. Rev. D51, (1995), 4474.

[24] I. Aitchison and C.M. Fraser, Phys. Lett. B146, (1984) 63; Phys. Rev. D31, (1985), 2605; C.M. Fraser, Z. Phys. C28, (1985), 101.

[25] T.R. Morris and M.D. Turner, Nucl. Phys. B 509 [FS], (1998), 637.

[26] G.R. Golner and E.K. Riedel, Phys. Rev. Lett. bf 34, (1975) 856; Phys. Lett. bf A58, (1976) 11; E.K. Riedel, G.R. Golner and K.E. Newman, Ann. Phys. 161, (1985), 178.

[27] J. Comellas and A. Travesset, Nucl. Phys. B 498, (1997), 539; J. Comellas, Nucl. Phys. B 509, (1998), 662; R. D. Ball et al, Phys. Lett. B347, (1995) 80 .

[28] B.G. Nickel and J.J. Rehr, J. Stat. Phys. 61, (1990), 1.

[29] P. Butera and M. Comi, Phys. Rev. B56, (1997), 8212.

[30] C.F. Baillie, R. Gupta,K.A. Hawick and G.S. Pawley, Phys. Rev. B45, (1992), 10438, and references therein.

[31] H.G. Ballesteros, L.A. Fernandez, V. Martin-Mayor, A. Munoz Sudupe, Phys. Lett. B387, (1996), 125.

[32] J. Zinn-Justin, Quantum Field Theory and Critical Phenomena, (3rd ed., Oxford University Press, 1996).

[33] M. Hasenbush, J. Phys. A32, (1999), 4851. 


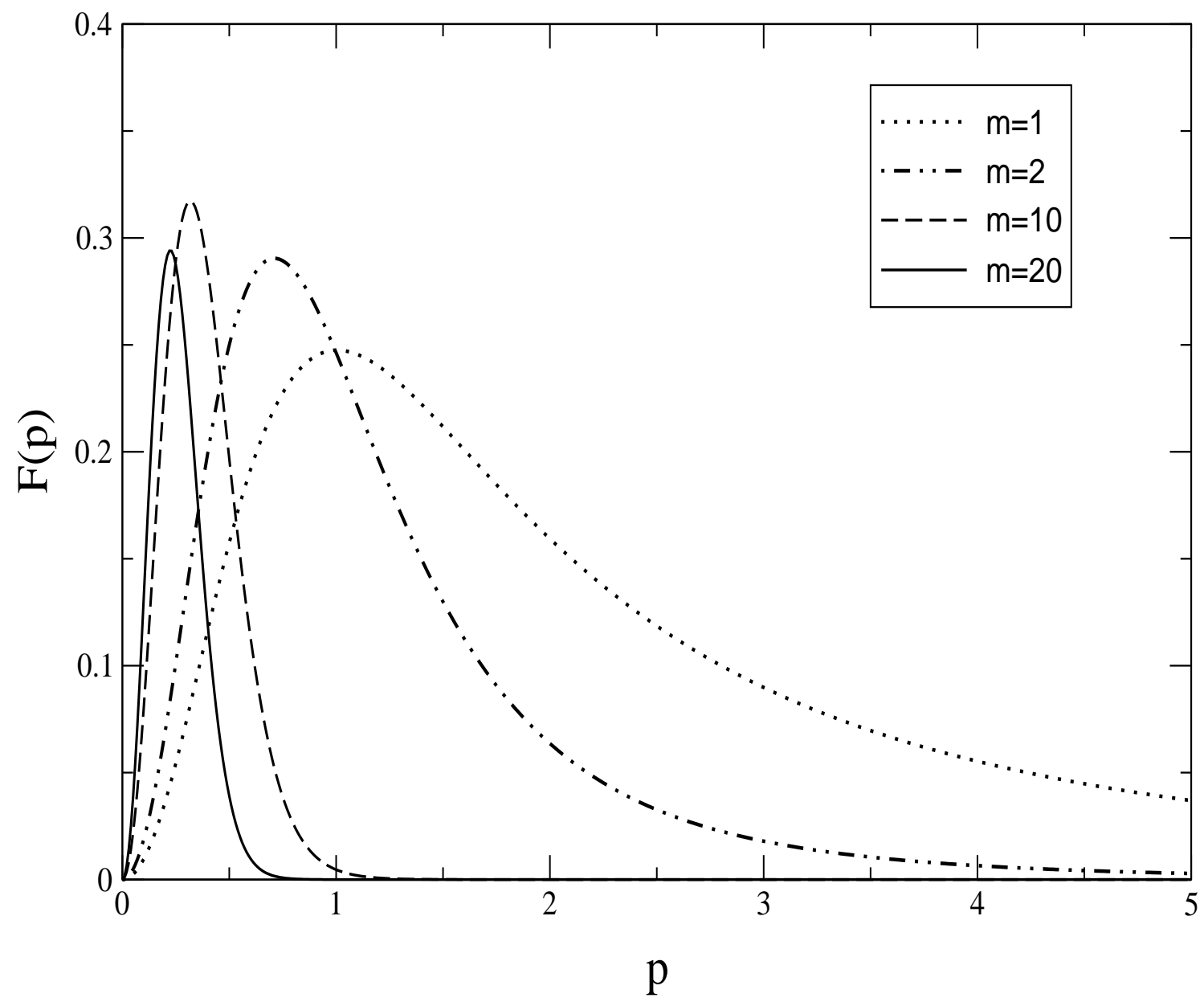

Figure 1: The function $F(p)$ (see text), plotted vs. $p$, for fixed $k=1, V^{\prime \prime}=0.01$ and $m=1,2,10,20$. 